\title{
Evaluation and Optimization of an Innovative Low-Cost Photovoltaic Solar Concentrator
}

\author{
Franco Cotana, Federico Rossi, and Andrea Nicolini \\ Department of Industrial Engineering, University of Perugia, Via G. Duranti 67, 06125 Perugia, Italy \\ Correspondence should be addressed to Andrea Nicolini, nicolini.unipg@ciriaf.it
}

Received 19 May 2011; Accepted 15 July 2011

Academic Editor: Gion Calzaferri

Copyright () 2011 Franco Cotana et al. This is an open access article distributed under the Creative Commons Attribution License, which permits unrestricted use, distribution, and reproduction in any medium, provided the original work is properly cited.

\begin{abstract}
Many researches showed that the cost of the energy produced by photovoltaic (PV) concentrators is strongly reduced with respect to flat panels, especially in those countries that have a high solar irradiation. The cost drop comes from the reduction of the expensive high-efficiency photovoltaic surface through the use of optical concentrators of the solar radiation. In this paper, an experimental innovative PV low-concentration system is analysed. Numerical simulations were performed to determine the possible reasons of energy losses in the prototype, primarily due to geometrical factors. In particular, the effect of the shadows produced from the mirrors on the prototype performances was analysed: shadows are often neglected in the design phase of such systems. The study demonstrates that shadows may affect the performances of a hypothetical optimized PV low-concentration system up to $15 \%$. Finally, an economical evaluation was carried out comparing the proposed optimized system to a traditional flat PV panel.
\end{abstract}

\section{Introduction}

Solar energy is a huge free resource for the world energetic demand. Direct conversion of sunlight into electricity in PV cells is one of the main solar active technologies, the two others being concentrating solar power (CSP) and solar thermal collectors for heating and cooling [1]. PV cells provide $0.1 \%$ of the global electricity generation and are projected to provide $5 \%$ of global electricity consumption in 2030 , rising up to $11 \%$ in 2050 [2]. Many researches showed that the cost of the energy produced by traditional flat PV panels may be reduced by PV concentrators [3-5]. Since semiconductor material is the most expensive part of the PV system and PV cells can theoretically produce more energy if exposed to higher sunlight fluxes, PV concentrators are based on focusing a large amount of solar radiation on a small surface of high-efficiency PV cells. Different concentrator systems were proposed in the past years [3]: however, their economic suitability was limited by the low availability of PV cells able to work in concentrating conditions, the problems in implementing efficient PV cooling systems, the necessity of efficient and cheap solar tracking systems. Nowadays, these technologies reached, for different reasons, a good development level. However, medium and high PV concentration systems (resp. with $20-500 \mathrm{x}$ and over 500x concentration levels) need expensive multijunction PV cells which are characterized by high efficiency but a performance drop when they are installed in hot environments (such as deserts) $[6,7]$. Furthermore, they need parabolic optical systems or Fresnel lens systems for the concentration, while high performances have also to be given by the cooling system due to the high concentration ratios. These considerations give an interesting economic value to low-concentration PV systems which are characterized by a concentration ratio lower than 20x. This paper deals with a theoretical analysis of the performances and the problems which may characterize the design of an innovative low-concentration photovoltaic (PV) prototype. The prototype is made of flat mirrors, which constitute the optical low-concentration system, and a monocrystalline PV panel. The movement of the mirrors, in order to follow the sun path, is run by asynchronous motors. In a previous work, experimental data were taken from the prototype in order to determine the best cooling system since the temperature increase drops off the efficiency of the PV panel [8]: a theoretical analysis showed that the best option is the natural convection with a finned surface. 
The specific task addressed by this work is the evaluation of the possible reasons that may affect the prototype correct working conditions in order to optimize its performances. In particular, the evaluation of energy losses due to the shadows produced from the mirrors was determined by numerical simulations. Results showed that a correct design of this kind of concentrators has not to neglect the shadow influence on the system. Thus, the analysis results will allow calculating a corrective angle for mirrors' rotation in case that the reflected rays, because of the shadows produced from the mirrors among them, do not hit the PV panel. Furthermore, a technical-economic evaluation was made in order to compare the cost trend of the proposed systems to the one of traditional flat PV systems.

\section{The Planar Low-Concentration Prototype}

An experimental innovative PV low-concentration system was proposed and built. The incident radiation is concentrated on a PV panel by means of a set of flat mirrors. The mirrors, appropriately oriented with respect to the PV receiver panel, simulate the surface of a parabolic concentrator (see Figure 1). This solution allows a double simplification with respect to the parabolic concentrators both in the manufacturing phase and the working phase of the facility, also because the mirrors are characterized only by one rotational axis.

An electronic system for tracking management rotates the mirrors around the rotational axis, in such a way that the solar radiation is always concentrated on the PV receiver panel during the sun daily movement. The receiver panel is made of common PV cells of monocrystalline silicon that constitutes a mature technology. Monocrystalline silicon cells are suitable for low-concentration levels (lower than $20 \mathrm{x}$ ), enabling a remarkable reduction of the required collector surface, but keeping the advantages of a consolidated technology $[9,10]$. The proposed technology has some advantages with respect to the traditional flat PV panels and to the high-concentration systems. With respect to the flat panels, low-concentration system allows more produced energy with the same number of PV cells or less number of PV cells for producing the same amount of energy. Furthermore, the efficiency of the PV cells rises with the increase in irradiation whenever they are cooled. Regarding the highconcentration systems, low-concentration allows the use of traditional PV cells in spite of multijunction PV cells (particularly complex and expensive). Theoretic experimental studies were developed in order to evaluate the best solution for heat dissipation. It was found that the optimal cooling system for the prototype was the natural convection [8]. Thus, the proposed system may be considered as divided into two subsystems:

(i) tracking and reflection subsystem;

(ii) picking and heat dissipation subsystem.

2.1. Tracking and Reflection Subsystem. The system is made of a series of rotating mirrors and a PV panel. The axes of the mirrors are parallel to each other and lie in a plane parallel

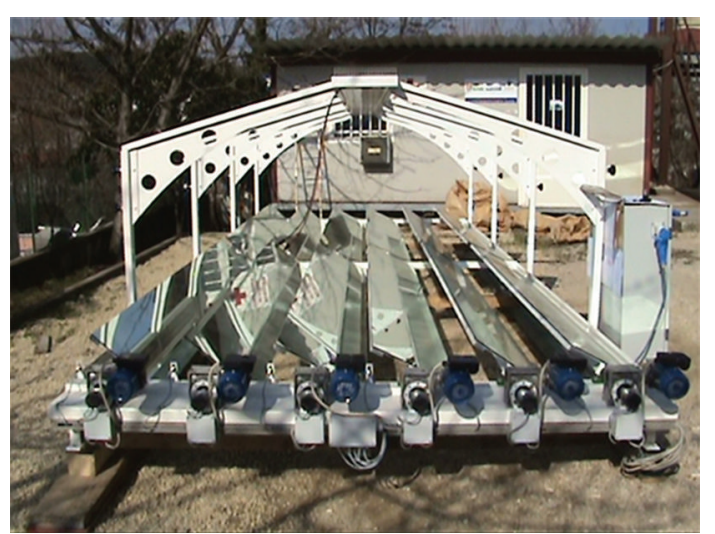

FIGURE 1: The innovative PV low-concentration prototype.

to the PV panel. The optimization of the geometric configuration was performed by means of numerical simulations developed by a MATLAB code. As the place of interest was considered the city of Perugia (lat. $43^{\circ} 6^{\prime}$, long. $12^{\circ} 23^{\prime}$ ), in Italy; in order to study the energetic effect of the sun, the incident rays were considered parallel among them. Moreover, each mirror was divided into rectangular blocks, and the centre of each block was considered as the source of the reflecting ray carrying the energy for the whole block. By considering classical relations for sun position and movement $[11,12]$, the developed algorithm takes into account the following conditions.

(i) Sunlight direction: for a given time in a given day of the year it finds out the direction of the sun, assuming all the rays parallel to each other.

(ii) Opacity conditions: for a given angle position of each mirror, it establishes if the rays hit the reflecting side or the opaque one. If they hit the opaque side, they certainly cannot hit the PV panel.

(iii) Rising condition: for a given angle position of each mirror, it establishes if the rays reflecting from the mirror rise or not (rising condition). If not they certainly cannot hit the PV panel.

(iv) Block energetic effect: it computes the energetic effect of a single block of the mirror on the PV panel.

(v) Panel shadow: for each mirror it finds out which blocks are in the shade of the panel.

For simplicity, in the first design phase, the possible influence of the shadows among the mirrors on the system performance was neglected.

In order to find the best configuration from an energetic point of view, different configurations of the system were simulated varying some geometric parameters (the dimensions of the mirrors, and the PV panel, the orientation of the PV panel, the number of the mirrors, the distance between adjacent mirrors, and the height of the PV panel above the mirrors' plane). 
Simulations are allowed determining that:

(i) the optimum orientation is north-south direction so that the longitudinal axes of the mirrors are placed in such direction;

(ii) the optimum width value of the mirrors is 1.35 times the width of the PV panel $\left(e_{m}=1.35 e_{c}\right)$. This helps to compensate the losses due to the null inclination of the mirrors with respect to the ground, because of constructive simplicity. The following construction values were chosen: $e_{m}=0.39 \mathrm{~m}, e_{c}=0.29 \mathrm{~m}$. The gap between adjacent mirrors was chosen as $0.01 \mathrm{~m}$;

(iii) the optimum length of each mirror should be 1.5 times the length of the panel: $l_{m}=1.5 l_{c}$. However, in order to reduce the prototype dimensions and obtain commercialization possibilities for the proposed system, the following length values were chosen: $l_{m}=$ $6.355 \mathrm{~m}, l_{c}=5.400 \mathrm{~m}$; thus, a PV panel able to produce about $200 \mathrm{~W}$ as single flat panel was used;

(iv) the optimum number of mirrors is six, compatible with the increase in electric current due to the greater incident radiation on the PV panel;

(v) the optimum value of the height of the panel with respect to the mirrors is 0.5 times the total width of the mirrors: $h=0.5 \cdot 6 \cdot e_{m}(h=1.17 \mathrm{~m})$.

Each mirror has its own configuration in order to follow the sun movement and focus the income radiation on the PV cells. For this reason, each mirror is rotated by its own electric motor which is managed by a programmable logic controller (PLC). The front view of the installation is shown in Figure 2: it is shown the tracking system for the mirrors which are laid on a metallic structure, parallel to the PV cells, placed above. The algorithm for the mirror movement calculates, for each mirror, the rotational angle needed for the reflected rays to hit the PV receiver. The movement of the mirrors is done around a single rotational axis at the longitudinal direction of the mirror (north-south direction).

The rotation of each mirror, " $x$," is given by the following (see also Figure 3):

$$
\begin{gathered}
x=\alpha_{y z}+\alpha_{i}-\frac{\pi}{2}, \\
\alpha_{y z}+\alpha_{i}+r+c=\pi, \\
c=\arctan \left(\frac{A}{B}\right),
\end{gathered}
$$

where " $\alpha_{i}$ " is the incident angle of the solar ray with respect to the normal of the rotated mirror, " $r$ " is the reflection angle with respect to the normal of the mirror, " $c$ " is the angle between the PV panel and the mirror, " $A$ " is the height of the PV panel, and " $B$ " is the distance of the mirror axis to the centre of the facility.

By the Snell law, it is obtained that $\alpha_{i}=r$, resulting that

$$
\alpha_{i}=\frac{\pi-c-\alpha_{y z}}{2}
$$

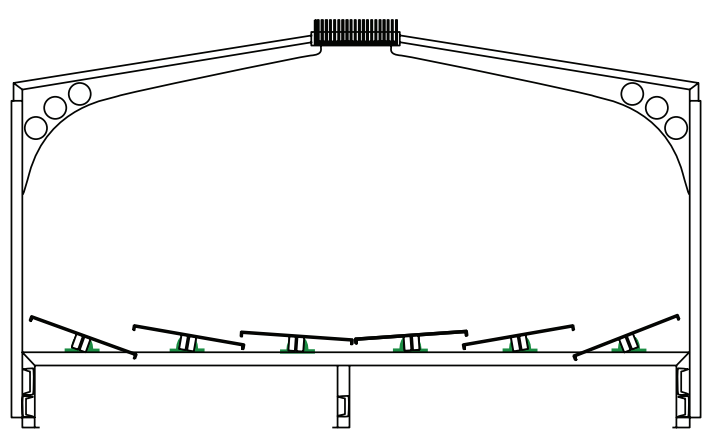

FIGURE 2: Frontal view of the low-concentration prototype.

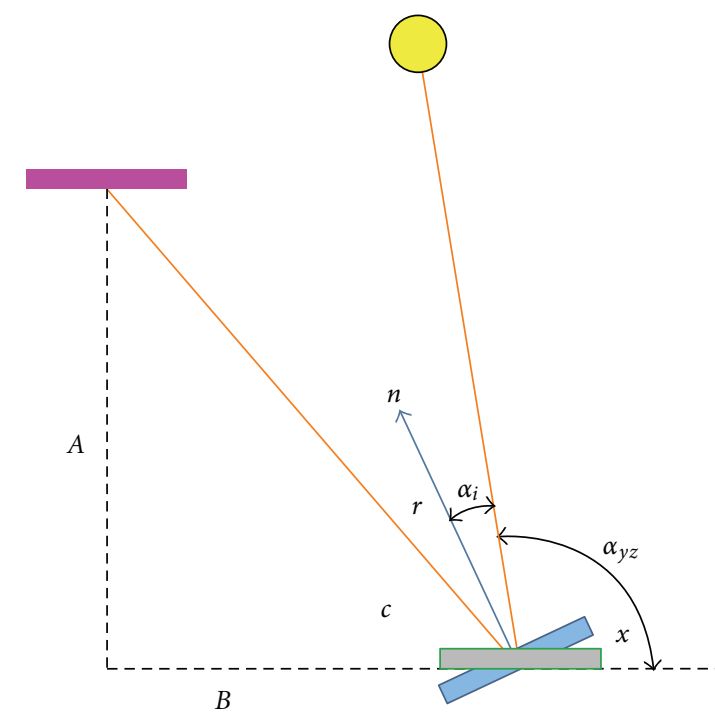

FIGURE 3: Rotation of a mirror and its configuration angles.

Thus

$$
x=\frac{\alpha_{y z}-c}{2} .
$$

The rotation of each mirror (for which $c$ is fixed) is, then, computed for each time step from the projection of the solar height on the $Y Z$ plane. The algorithm is implemented in a PLC that controls the prototype mirrors, and its efficiency has already been verified.

2.2. Picking and Heat Dissipation Subsystem. This subsystem is basically composed of the upper part of the structure, where the PV panel picks up the incoming radiation reflected by the mirrors and the finned surface is in charge of releasing the heat from the PV cells (see Figure 4).

\section{A Simplified Model for the Prototype Analysis}

A simplified 2D model for the low-concentration system was proposed: the model allows a simplified analysis of the performances of the prototype (differently from the complex algorithm which is implemented on the prototype and was used for the preliminary design) and the evaluation of 


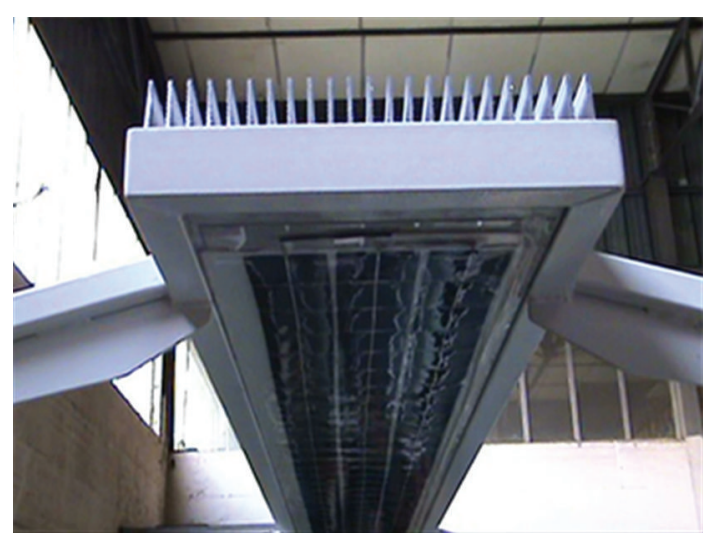

Figure 4: Detail of the PV panel and the finned surface.

the energy losses due to geometrical reasons (the reflected energy flux not hitting the PV panel). Thus, it may be easily used to optimize the proposed system. It is based on the following relation that is to be verified to determine if energy losses occur:

$$
e_{m} \cdot \cos \alpha_{i} \leq e_{c} \cdot \sin c
$$

where

(i) $e_{c} \cdot \sin c$ is the projection of the receiver panel width on the perpendicular plane to the panel-mirror connection line;

(ii) $e_{m} \cdot \cos \alpha_{i}$ is the projection of the side of the mirror on the perpendicular plane to the panel-mirror connection line.

In order to verify this condition for the proposed configuration, the three mirrors on the left hand side (called mirrors 1,2 , and 3 ) and the mirrors on the right hand side (called mirrors 4, 5, and 6) should be separately analysed. Furthermore, morning and afternoon conditions should be separately considered. Thus, four different cases were analysed.

3.1. Case 1 (mirrors on the left side, morning). From Figure 5, the following relation is obtained:

$$
\alpha_{i}=\frac{c}{2}-\frac{\alpha_{y z}}{2}
$$

Thus, (4) may be written as

$$
e_{m} \cos \left(\frac{c}{2}-\frac{\alpha_{y z}}{2}\right) \leq e_{c} \sin c
$$

From (6), an important design parameter (called ratio) is determined:

$$
\text { ratio }=\frac{e_{c} \sin c}{e_{m} \cos \left(c / 2-\alpha_{y z} / 2\right)}
$$

By this parameter, the available area from the PV panel is compared to the one reflected from the mirrors.

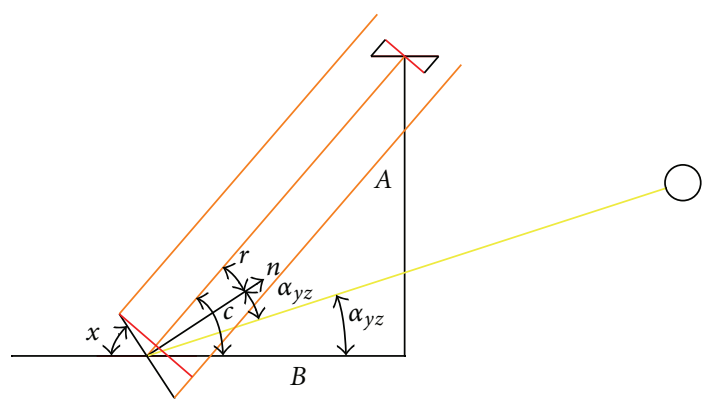

Figure 5: Case 1 configuration.

3.2. Case 2 (mirrors on the right side, morning). In a way close to Case 1 , the following expression for ratio is obtained:

$$
\text { ratio }=\frac{e_{c} \sin c}{e_{m} \cos \left(\pi / 2-c / 2-\alpha_{y z} / 2\right)}
$$

3.3. Case 3 (mirrors on the left side, afternoon). From Figure 6, the following relation is obtained:

$$
e_{m} \cos \left(\frac{\alpha_{y z}}{2}-\frac{c}{2}\right) \leq e_{c} \sin c
$$

From (9), ratio is determined as follows:

$$
\text { ratio }=\frac{e_{c} \sin c}{e_{m} \cos \left(\alpha_{y z} / 2-c / 2\right)}
$$

3.4. Case 4 (mirrors on the right side, afternoon). In a way close to the previous cases, the following equation is obtained:

$$
\text { ratio }=\frac{e_{c} \sin c}{e_{m} \cos \left(\alpha_{y z} / 2+c / 2-\pi / 2\right)}
$$

3.5. The Meaning of the Parameter "Ratio". Let us explain the meaning of the different values the ratio can take. If ratio is between zero and one, this means that the projected area from the mirror is bigger than the available one from the PV panel; thus, the whole PV panel surface is hit by sunlight but not in an effective way because the facility is losing solar energy that could be transduced in electrical energy. If ratio is zero, there is no sunlight hitting the PV panel (no electrical energy produced). When ratio is greater than one, PV panel area is bigger than the one reflected from the mirrors. This is the worst case in which the installation can be found because only some of the PV cells are hit by the sunlight; the global produced current will be highly limited.

3.6. Simulation Results. Ratio values were calculated in order to analyse the general behaviour of each mirror along the year. Classical equations for describing the sun position were used [11]. The found one-hour step ratio values for each mirror are reported in Figures 7, 8, 9, 10, 11, and 12. 


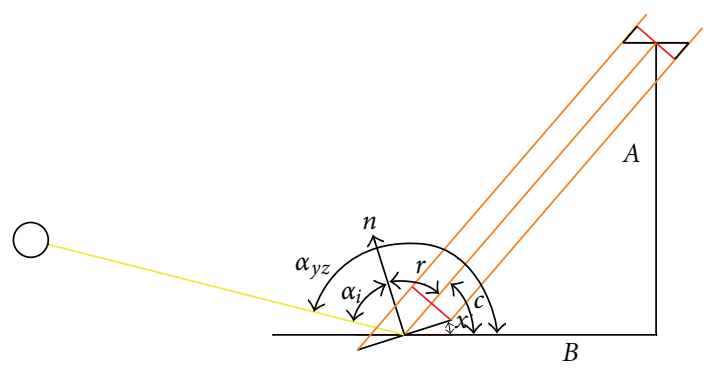

Figure 6: Case 3 configuration.

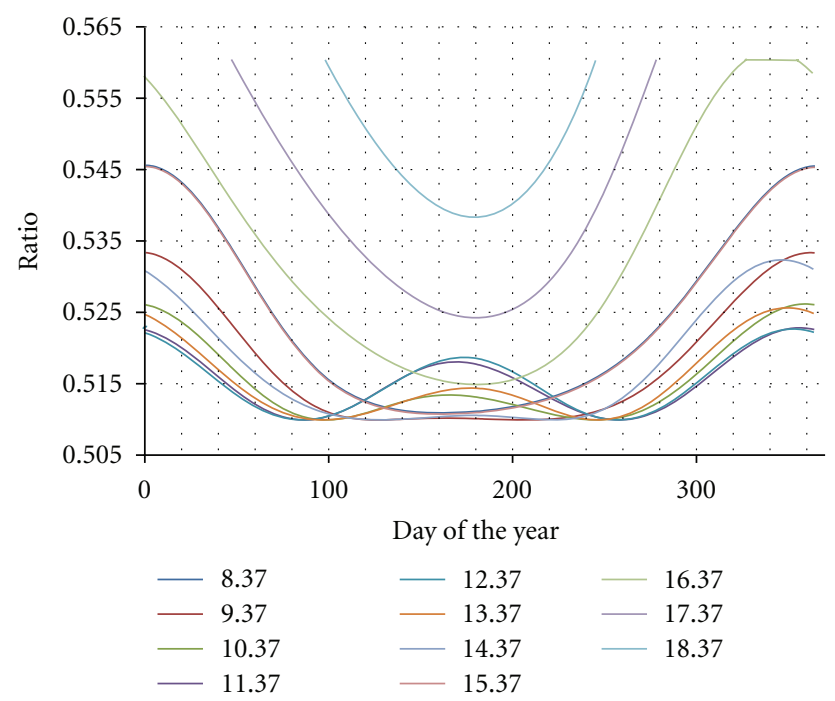

Figure 7: Ratio values of mirror 1 along the year (one-hour steps).

As shown in Figures 7-12, the general behaviour of the mirrors is almost the same along the year but, depending on the time of the day, the ratio value changes. For all the mirrors, ratio is higher at 5 and $6 \mathrm{pm}$ in the afternoon. By the contrary, it takes the lower values at midday; it means that when there is the higher irradiation of the day, the configuration of the facility cannot achieve the maximum of it. The proposed model was validated by geometrical measurements on the prototype, made in the four days of the year with more, average, or less sun irradiation of the year: the two equinoxes and the two solstices: measurements showed that the model was correctly satisfied for each one of the four days analysed resulting in a difference in the ratio values from the fourth decimal.

3.7. Annual Energy Calculation. In order to evaluate the total solar energy which hits the PV panel, ASHRAE clear-sky irradiation model was used [12]. Ratio values were used to evaluate the global energy which hits the PV panel by an implemented MATLAB code. Evaluation results give that the annual energy which hits the PV panel is $4195 \mathrm{kWh} / \mathrm{m}^{2}\left(\mathrm{~m}^{2}\right.$ refers to the PV panel surface). It is a reference value for the next research steps.

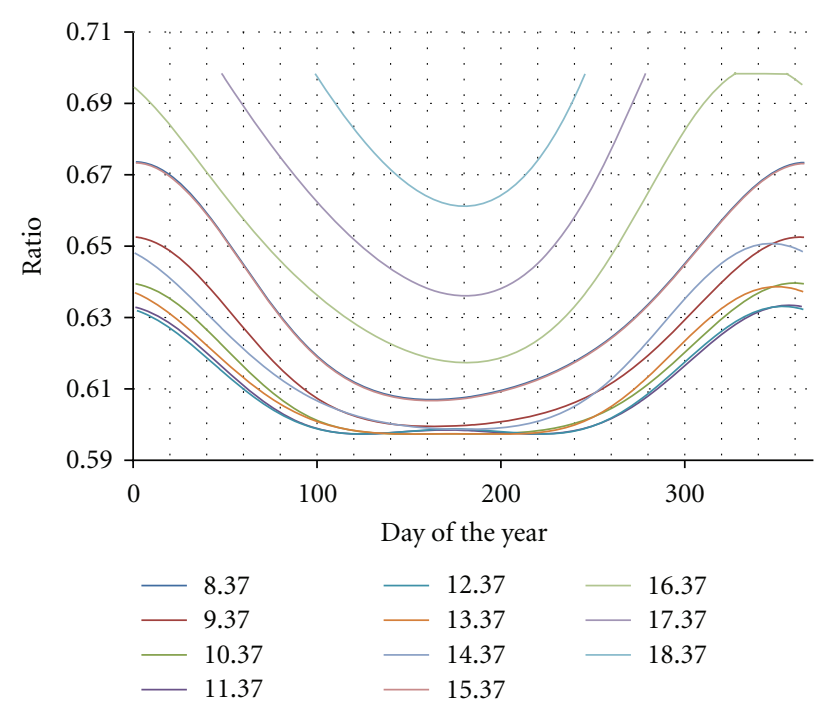

FIGURE 8: Ratio values of mirror 2 along the year (one-hour steps).

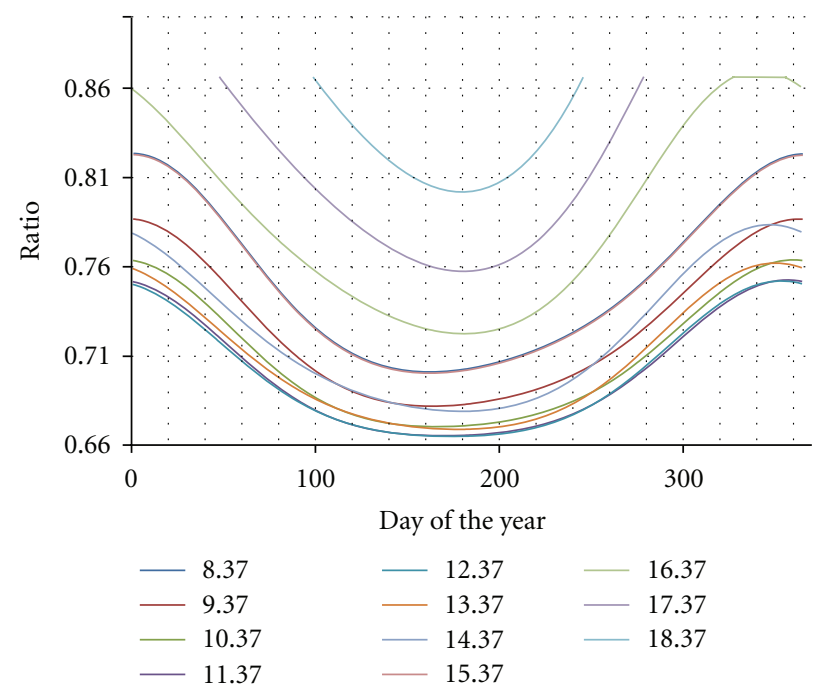

Figure 9: Ratio values of mirror 3 along the year (one-hour steps).

\section{System Optimization by the Evaluation of the Effect of Shadows Produced by the Mirrors among Them on the Facility Performances}

The effect of shadows produced by the mirrors among them on the concentrator performances was neglected in the preliminary design phase; the energy losses due to the shadows were determined by the proposed model in order to evaluate if the previous design hypothesis may be considered available. Due to the shadow creation or the interference of one mirror at the projected area, the resulting concentrated area could not match the PV panel. For this reason, depending on the new hitting area geometry, ratio may change with respect to the one given by (7), (8), (10), and (11). The following cases may occur. 


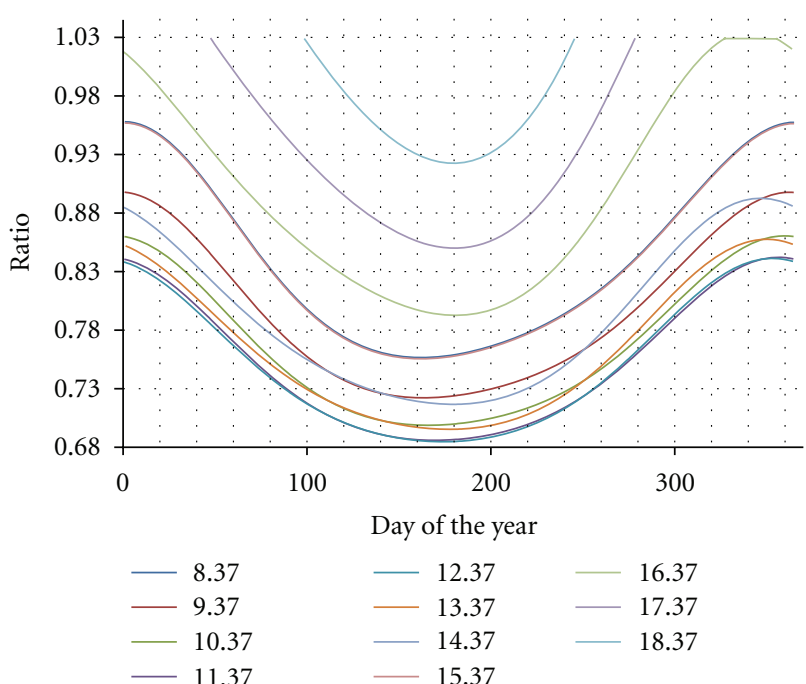

FIGURE 10: Ratio values of mirror 4 along the year (one-hour steps).

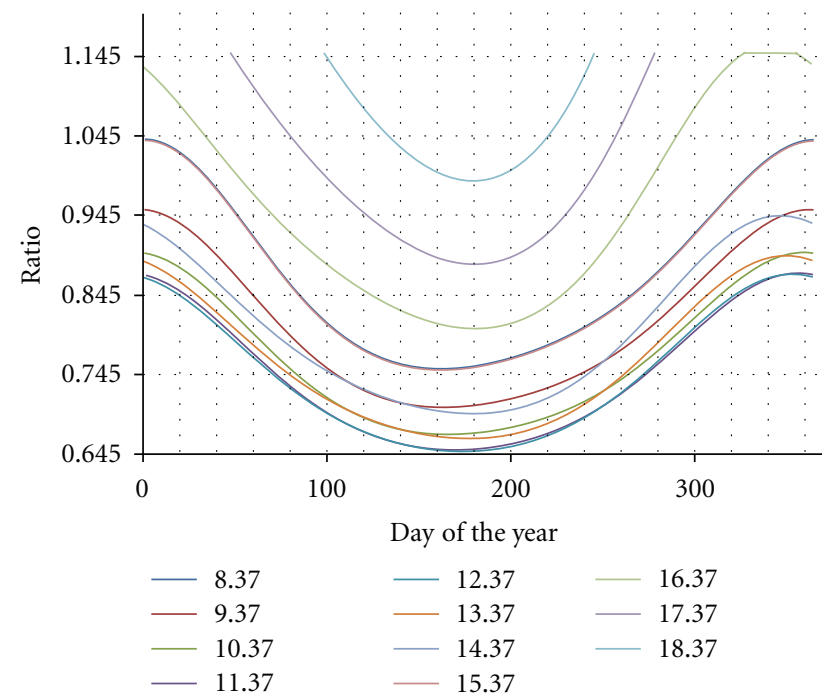

FIGURE 11: Ratio values of mirror 5 along the year (one-hour steps).

Case A. When both limits of the reflected area are out of the PV panel (see Figure 13), ratio is given by

$$
\text { ratio }=\frac{e_{c}}{\left|x_{\text {right }}-x_{\text {left }}\right|} .
$$

This is the same way as it was obtained without considering the shadow effect but, in this case, the coming sunrays have changed.

Case B. In this case, because of the shadows or the interference of other mirror, one of the borders of the reflected area, the left hand side one, cuts the panel (see Figure 14).

Thus, ratio is given by

$$
\text { ratio }=\frac{e_{c}}{\left|e_{c} / 2-x_{\mathrm{left}}\right|}
$$

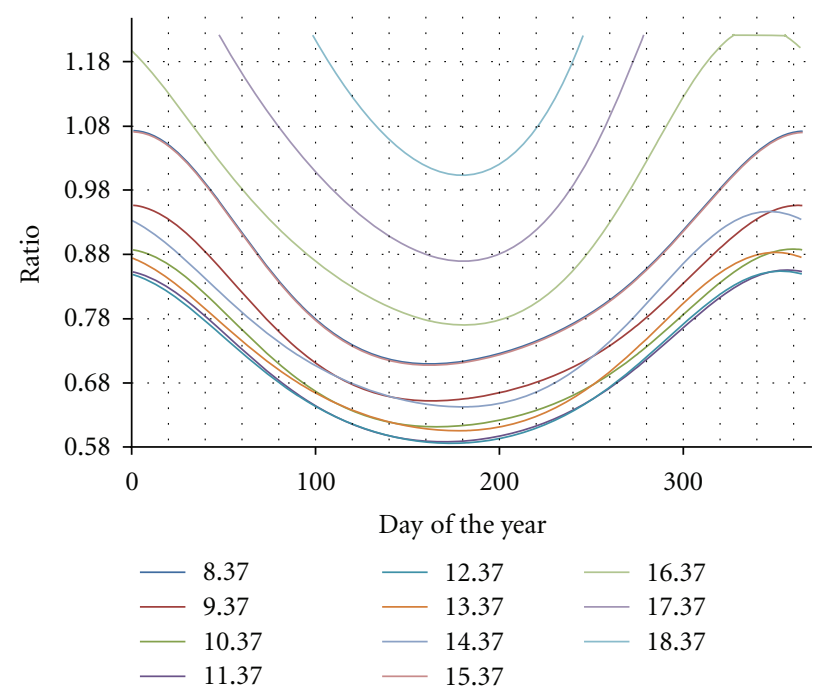

FIGURE 12: Ratio values of mirror 6 along the year (one-hour steps).

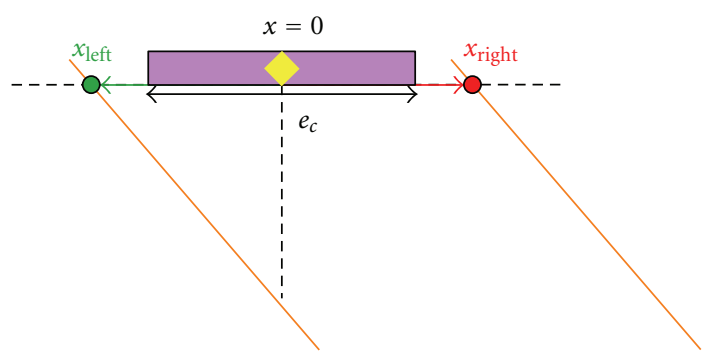

Figure 13: Ratio calculation by taking into account shadows. Case A configuration.

Case C. This situation is the same as in the previous case but with the right hand side limit of the reflected area. Ratio is given by

$$
\text { ratio }=\frac{e_{c}}{\left|e_{c} / 2+x_{\text {right }}\right|}
$$

Case D. If both bounds of the concentrated area are inside the PV panel, ratio is given by the same relation of Case A but the crossing points have changed:

$$
\text { ratio }=\frac{e_{c}}{\left|x_{\text {right }}-x_{\text {left }}\right|} .
$$

Case E. This case is split into two other cases since it corresponds to the situation in which none of the bounds hit the panel; thus, the whole reflected area is out of the PV panel. The reflected area can be at the right (see Figure 15) or at the left hand side of the PV panel.

For both cases, the reflected area from the mirrors does not match in any point the PV panel; thus, ratio is null.

4.1. Evaluation of Energy Losses due to the Shadows. Once ratio values have been computed for all the year, their changes with respect to the previous analysis (neglected shadows) 


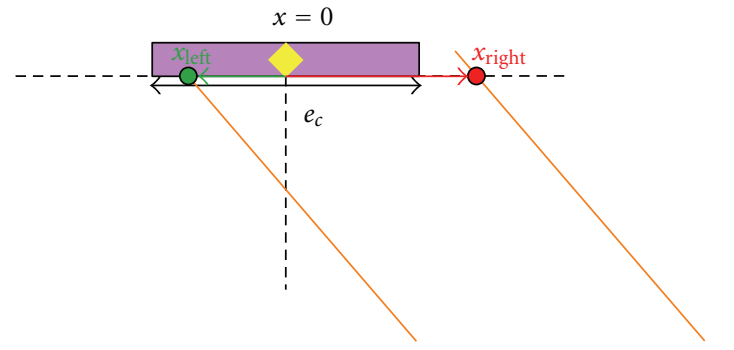

FIGURE 14: Ratio calculation by taking into account shadows. Case B configuration.

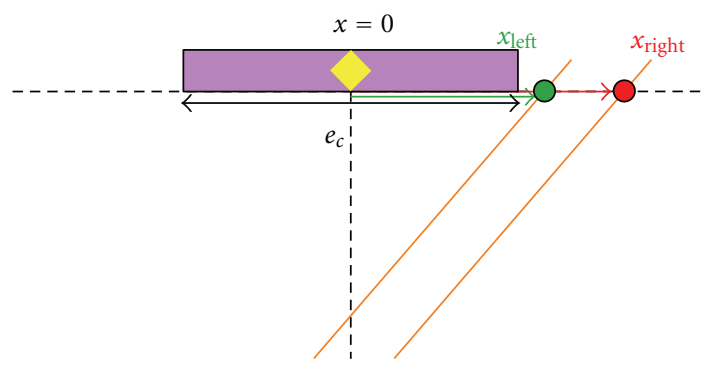

FIgURE 15: Ratio calculation by taking into account shadows. Case E configuration (reflected area at the right side of the PV panel).

have to be determined in order to evaluate the energy losses due to the shadows. If the ratio variation takes place early in the morning or late in the afternoon, when the solar irradiation is low, the effect of the shadows would not be so important. But, on the contrary, if this variation is at midday, when the solar irradiation is high, the shadow effect would be significant to be considered in the prototype design. Simulations were performed to evaluate the ratio values when the shadow effect is considered. Because of the way in which ratio has been defined, its value may vary drastically from zero to a great value. In order to explain the reason, it is made reference to Figure 15: shadows make the reflected light not to hit the panel at any point; for this reason, ratio is null. But, as the sun rises along the morning, the shadowed area is decreased and the left hand side border begins to move inside the PV panel area.

This is a Case B situation, and ratio is given by (see Figure 16 as reference scheme)

$$
\text { ratio }=\frac{e_{c}}{e_{m}} .
$$

Since the width of the PV panel is considerably bigger than the one given by the reflected radiation, ratio becomes huge when a few minutes before its value was zero. Very high ratio values are a bad condition for the system energy production as explained in Section 3.5. The results of the analysis performed for each mirror are reported in Figures 17-22. About mirror 1, as it can be seen in Figure 17, there are very high values at $8: 30 \mathrm{am}$, far away from the rest of the day. It only happens in days that belong to winter or autumn. Furthermore, the upper limit of ratios has risen

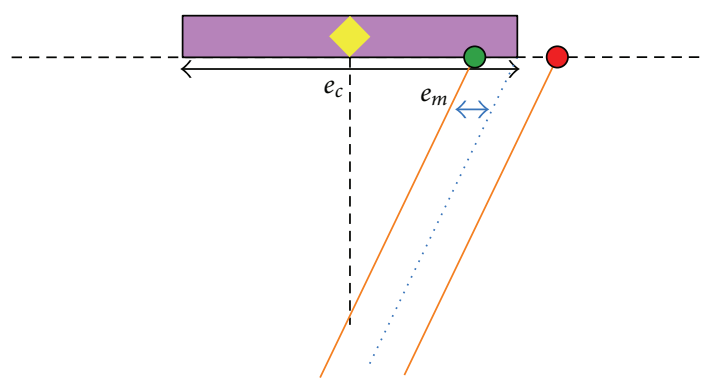

FIgURE 16: Changes in ratio value. Configuration which may give a very high ratio value.

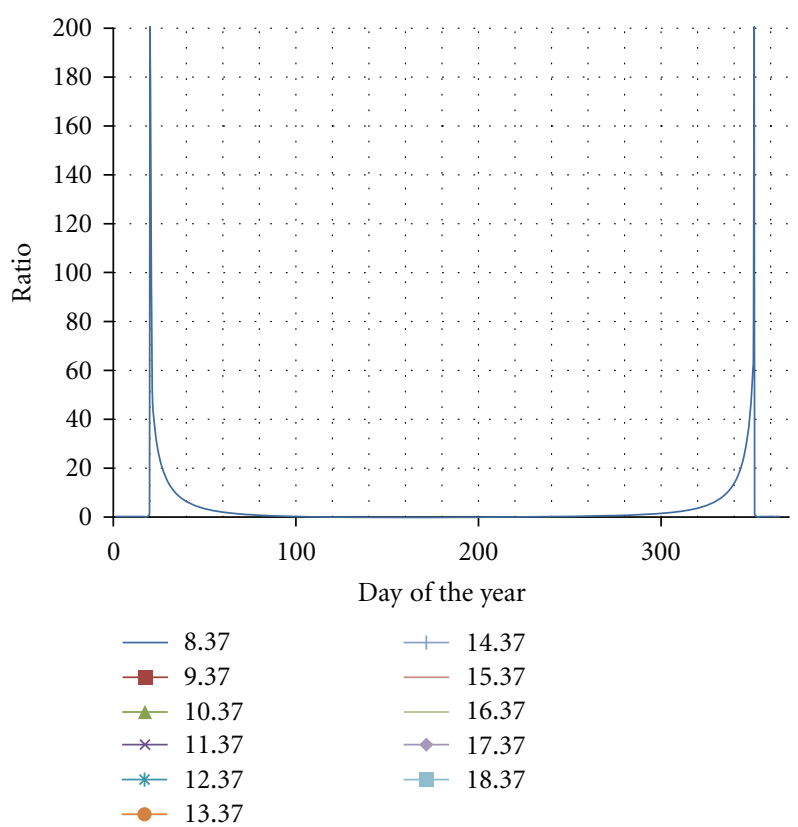

FIGURE 17: Ratio of mirror 1 along the year (shadows are considered).

considerably, not only for the curves in the early morning. All the curves moved upwards; the mean value of ratio for the whole year for the first mirror is around 0.97 ( 0.52 is the value of ratio by neglecting the shadows). It seems that ratio has been improved but really the cases in which it has increased a lot (because of the small PV panel surface covered by the concentrated sunlight) compensate the cases in which ratio has dropped. About mirror 2 (see Figure 18), many curves change their behavior but more significantly the ones which regard early morning or late afternoon. Just as happened for mirror 2, ratios of mirror 3 (see Figure 19) increased but only in a meaningful way for the moments in which the sun irradiation is not that important. Also for mirror 4 (see Figure 20), ratios arise with great values but only at late afternoon. Figure 21 shows results for mirror 5. In this case, even for the hours at midday, ratios increased a lot so the losses due to the shadows can be important enough to be considered in the prototype design. At last, for mirror 6 (see Figure 22), ratio curves moved upwards, increasing 


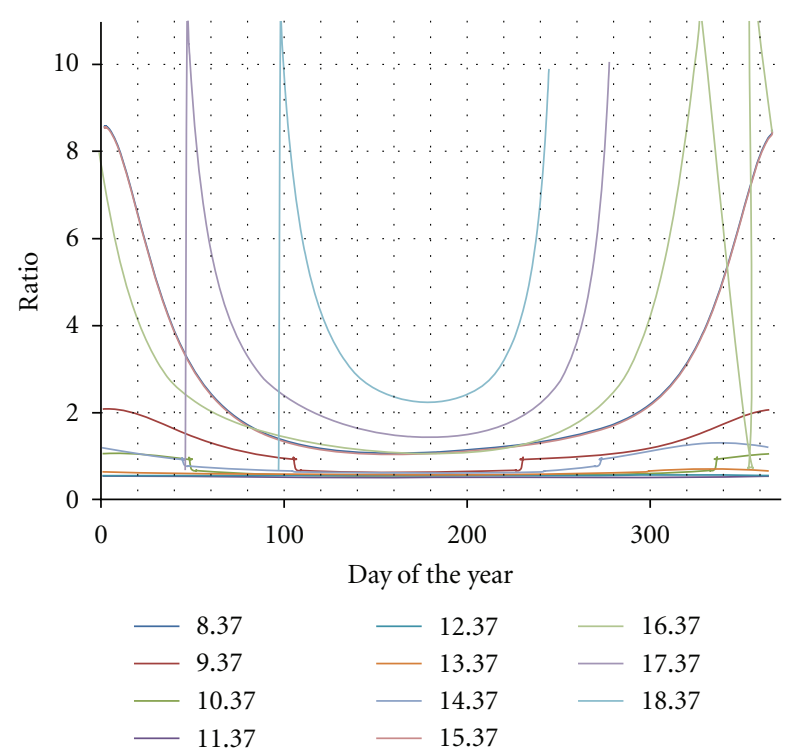

FIGURE 18: Ratio of mirror 2 along the year (shadows are considered).

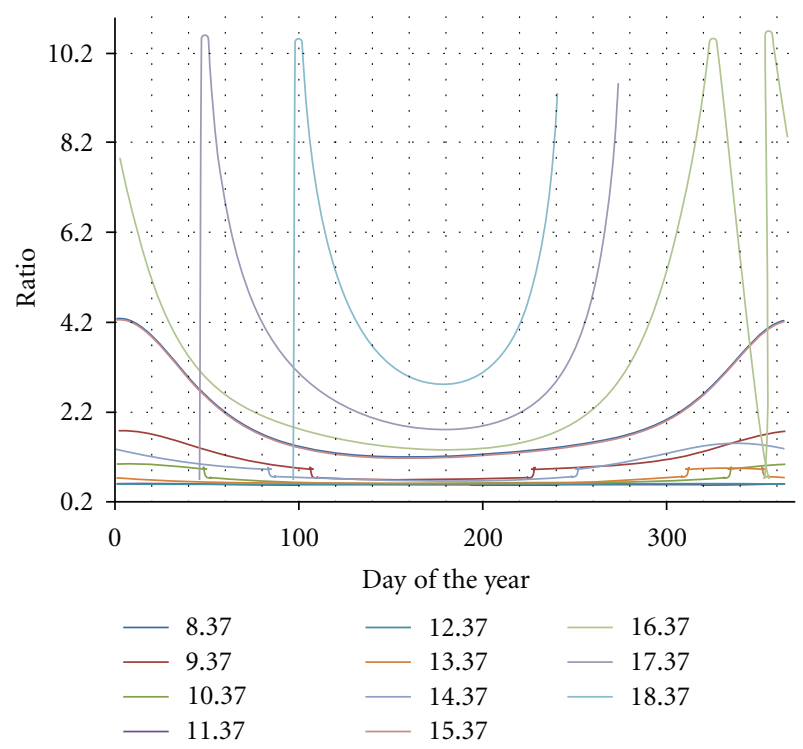

Figure 19: Ratio of mirror 3 along the year (shadows are considered).

the mean value for the whole year. But, this increase is not as great as for the other mirrors.

The total annual energy calculation was performed by Matlab calculations. As in the previous evaluation, when ratio arises from 1 to higher values, it was considered to limit the energetic capacity of the PV panel. It was found that the annual energy which hits the PV panel is $3596 \mathrm{kWh} / \mathrm{m}^{2}\left(\mathrm{~m}^{2}\right.$ refers to the PV panel surface) when shadows are considered: it is $14.3 \%$ lower than the one obtained by neglecting the shadows among the mirrors. This basically means that the shadows among the mirrors cannot be neglected in the design of solar concentrator systems as the one here

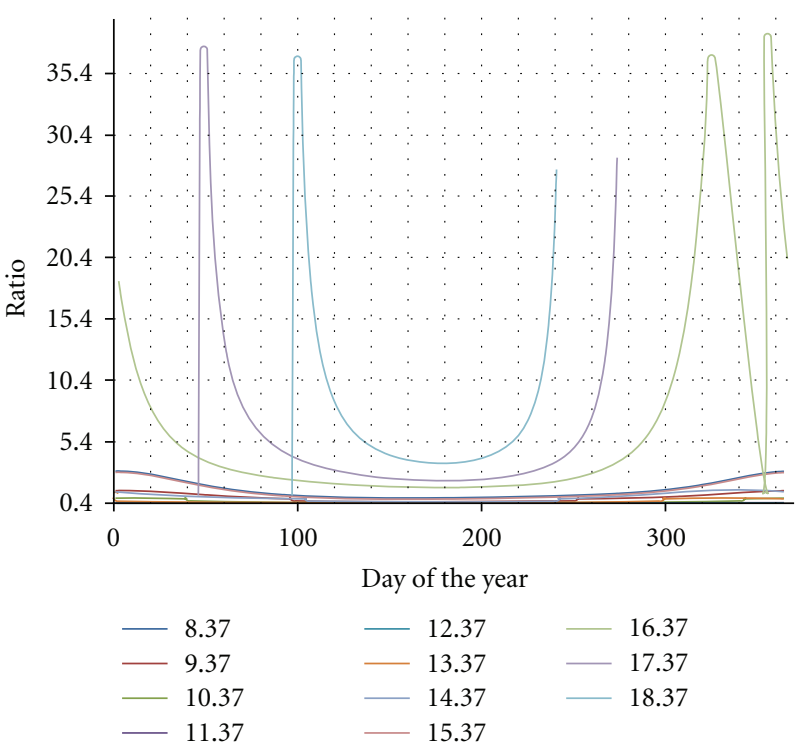

Figure 20: Ratio of mirror 4 along the year (shadows are considered).

proposed. Thus, an optimization process has to be obtained by changing the control system parameters: a solution could be rectifying the rotating angle of the mirrors in order to achieve a higher reflected area hitting the panel and reducing the number of ratios higher than one.

\section{Technical-Economic Comparison with Traditional Systems}

A technical-economic parameter (TEEC) was introduced to evaluate the future suitability of the proposed system:

$$
\begin{aligned}
& \text { TEEC } \\
& =\frac{\text { System cost }\left(€ / \mathrm{m}^{2}\right)}{\text { Annual Energy which hits the PV panel }\left(\mathrm{kWh} / \mathrm{m}^{2}\right)} .
\end{aligned}
$$

The cost and the computed annual energy are referred to a $1 \mathrm{~m}^{2}$ PV panel. A traditional PV panel at the Perugia location is hit by $1234 \mathrm{kWh} / \mathrm{m}^{2}$ for each $\mathrm{m}^{2}$ of $\mathrm{PV}$ panel (it was obtained by a numerical simulation based on the sun movement equations given in [11]). Thus, the proposed low-concentration system, if the shadows were neglected, would be characterized by an incident energy about 3.4 times higher than the traditional PV system. However, even if the proposed system was not optimized (shadows would produce a loss in the energy content which hits the PV panel), energy would be about 2.9 times than the one which hits the PV panel. The high variability of the financial incentives and demand of the traditional PV panels should induce a high uncertainty of their costs in the next years [13]. Furthermore, the proposed low-concentration system is made of materials (mainly steel) which may be characterized by a huge cost reduction when its components will be in the commercialization phase (now it is an experimental prototype). 


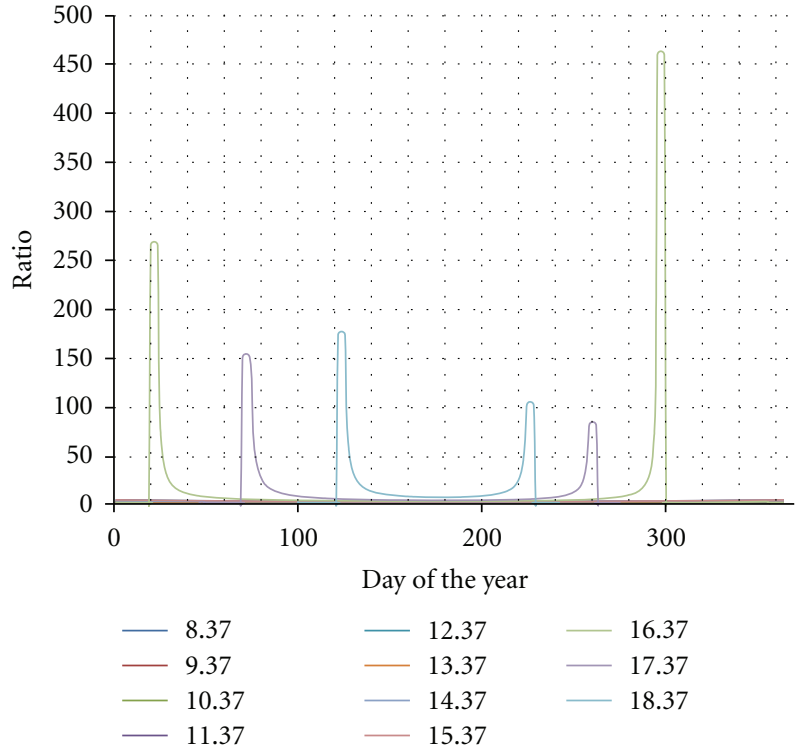

FIGURE 21: Ratio of mirror 5 along the year (shadows are considered).

Thus, if the costs of the steel structure components and the control system of the proposed low-concentration technology will reach about $€ 1000.00 / \mathrm{m}^{2}\left(\mathrm{~m}^{2}\right.$ of PV panel) in the commercialization phase, TEEC values for the analysed configurations would be the following [13] (referred to Perugia location and considering the actual price of PV panels):

$$
\begin{gathered}
\text { TEEC }_{\text {concentrator, no shadow }}=0.3179 \frac{€}{\mathrm{kWh}}, \\
\text { TEEC }_{\text {concentrator, shadow }}=0.3692 \frac{€}{\mathrm{kWh}}, \\
\text { TEEC }_{\text {flat PV panel }}=0.3647 \frac{€}{\mathrm{kWh}} .
\end{gathered}
$$

Equations (18) and (19) report respectively, TEEC values of the optimized proposed system (in which shadows are not considered) and the not-optimized proposed system. The evaluated cost of the proposed system involves the entire facility (PV panel, steel structure, inverter, control system). TEEC is evaluated by considering the actual price of monocrystalline PV panels and the mentioned cost of the steel structure and the control system in the commercialization phase [13]. Equation (20) reports the actual TEEC of the installation of a flat monocrystalline panel (it involves the cost of PV panel, installation structures, inverter). TEEC values show that only the proposed optimized system may be convenient with respect to the traditional PV systems. Furthermore, a technical-economic comparison between TEEC values of the proposed concentrator and traditional PV system is made by varying the cost of the PV panel. Figure 23 shows that the optimized concentrator is convenient with respect to the traditional PV system if the cost of the PV panel is higher than about $160 € / \mathrm{m}^{2}$. Besides, the not-optimized

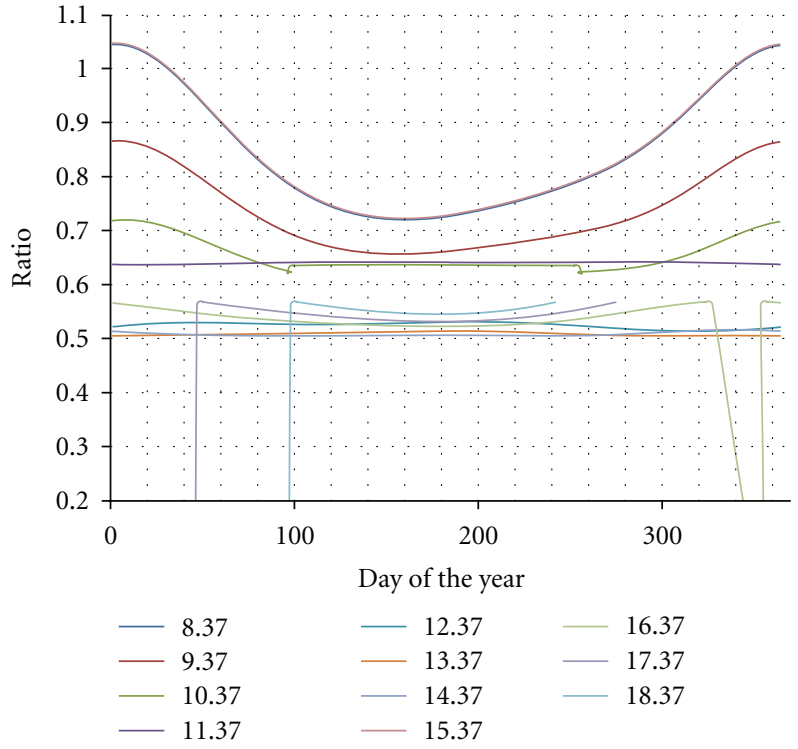

Figure 22: Ratio of mirror 6 along the year (shadows are considered).

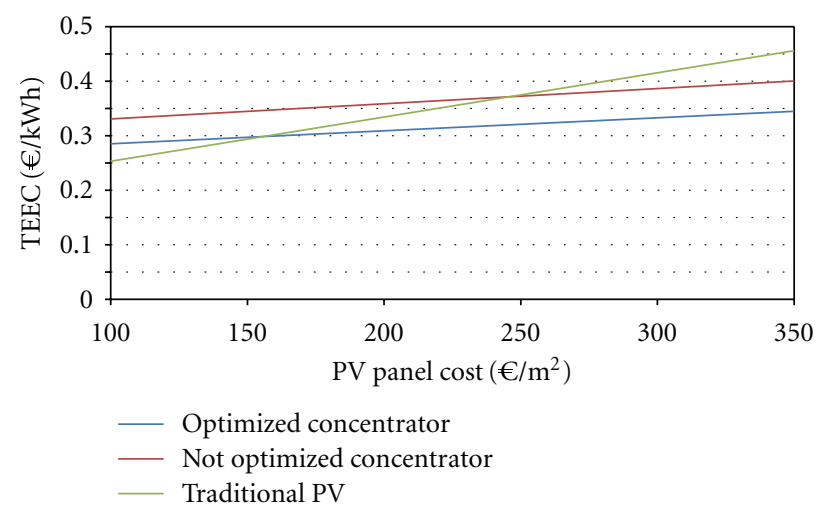

Figure 23: Comparison among TEEC values of the proposed concentrator (not-optimized and optimized) and the traditional PV system. The comparison is made versus the cost of a monocrystalline PV panel.

concentrator is convenient with respect to the traditional PV system if the cost of the PV panel is higher than about $250 € / \mathrm{m}^{2}$.

\section{Conclusions}

In this paper, an evaluation of the energy losses of a planar PV low-concentration prototype due to the shadows among the mirrors was made. The first step was the development of a mathematical model by which a ratio of the reflected sunlight by each mirror on the PV panel is achieved: a ratio parameter was introduced to evaluate the energetic performances of the proposed system. It was concluded that ratios bigger than one are undesirable and less convenient than ratios smaller than one, since the electric current is limited by the not matched PV panel area. Due to its geometrical configuration, 
the overall mean value for the ratio is about 0.75 , which is not a bad value, considering the ratio meaning as a kind of efficiency of the reflected sunlight on the PV panel. It should be taken into consideration that this value is affected also by the values that are bigger than one. Furthermore, the study of the importance of the shadows in the general performance of the facility was performed in order to optimize the system performances. It was defined that shadows cannot be neglected in the prototype design: the total energy achieved considering the shadow interaction was $14.3 \%$ lower than without including them in the design phase. Finally, a technical-economic evaluation was made in order to define the commercialization possibilities of the proposed system: it was shown that the optimized concentrator is convenient with respect to the traditional PV system if the cost of the PV panel is higher than about $160 € / \mathrm{m}^{2}$.

\section{References}

[1] A. C. Oliveira, "The energy shift: towards a renewable future," International Journal of Low Carbon Technologies, vol. 2, no. 3, pp. 289-299, 2007.

[2] International Energy Agency (IEA), “Technology roadmap. Solar photovoltaic energy," PVPS Annual Report, 2009.

[3] R. M. Swanson, "The Promise of concentrators," Progress in Photovoltaics: Research and Applications, vol. 8, no. 1, pp. 93111,2000

[4] S. Kurtz, "Opportunities and challenges for development of a mature concentrating photovoltaic power industry," Tech. Rep., National Renewable Energy Laboratory, 2009.

[5] A. K. M. Zakzouk, A. Mujahid, and M. S. El-Shobokshy, "Performance evaluation of photovoltaic silicon cells under concentrated sunlight," in Proceedings of the IEEE, vol. 131, no. 2, part 1, 1994.

[6] R. McConnell and M. Symko-Davies, "Multijunction photovoltaic technologies for high-performance concentrators," in Proceedings of the 4th IEEE World Conference on Photovoltaic Energy Conversion (WCPEC-4 '06), pp. 733-736, May 2006.

[7] H. Cotal, C. Fetzer, J. Boisvert et al., "III-V multijunction solar cells for concentrating photovoltaics," Energy and Environmental Science, vol. 2, pp. 174-192, 2009.

[8] F. Rossi, A. Nicolini, B. Castellani, and M. Giuliobello, "Scambio termico in convenzione naturale/forzata per celle fotovoltaiche a media concentrazione," in Proceedings of the 10th Congresso Nazionale CIRIAF, Perugia, Italy, 2010.

[9] F. Reis, M. C. Brito, V. Corregidor, J. Wemans, and G. Sorasio, "Modeling the performance of low concentration photovoltaic systems," Solar Energy Materials \& Solar Cells, vol. 94, no. 7, pp. 1222-1226, 2010.

[10] D. Walter, V. Everett, A. Blakers et al., "A 20-sun hybrid PVthermal linear micro-concentrator system for urban rooftop applications," in Proceedings of the 35th IEEE Photovoltaic Specialists Conference (PVSC '10), pp. 831-836, June 2010.

[11] M. Iqbal, An Introduction to Solar Radiation, Academic Press, New York, NY, USA, 1983.

[12] American Society and Heating and Refrigerating and Airconditioning Engineers (ASHRAE), Handbook of Fundamentals, 1972.

[13] U.S. Department of Energy, "2008 Solar Technologies Market Report," 2010. 


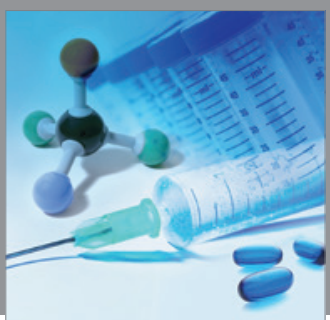

International Journal of

Medicinal Chemistry

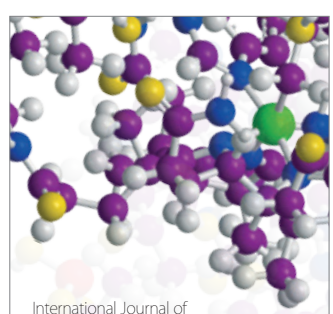

Carbohydrate Chemistry

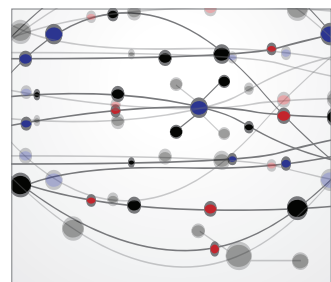

The Scientific World Journal
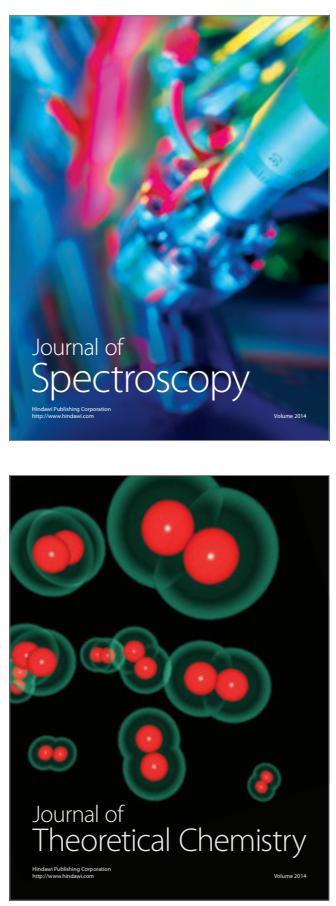
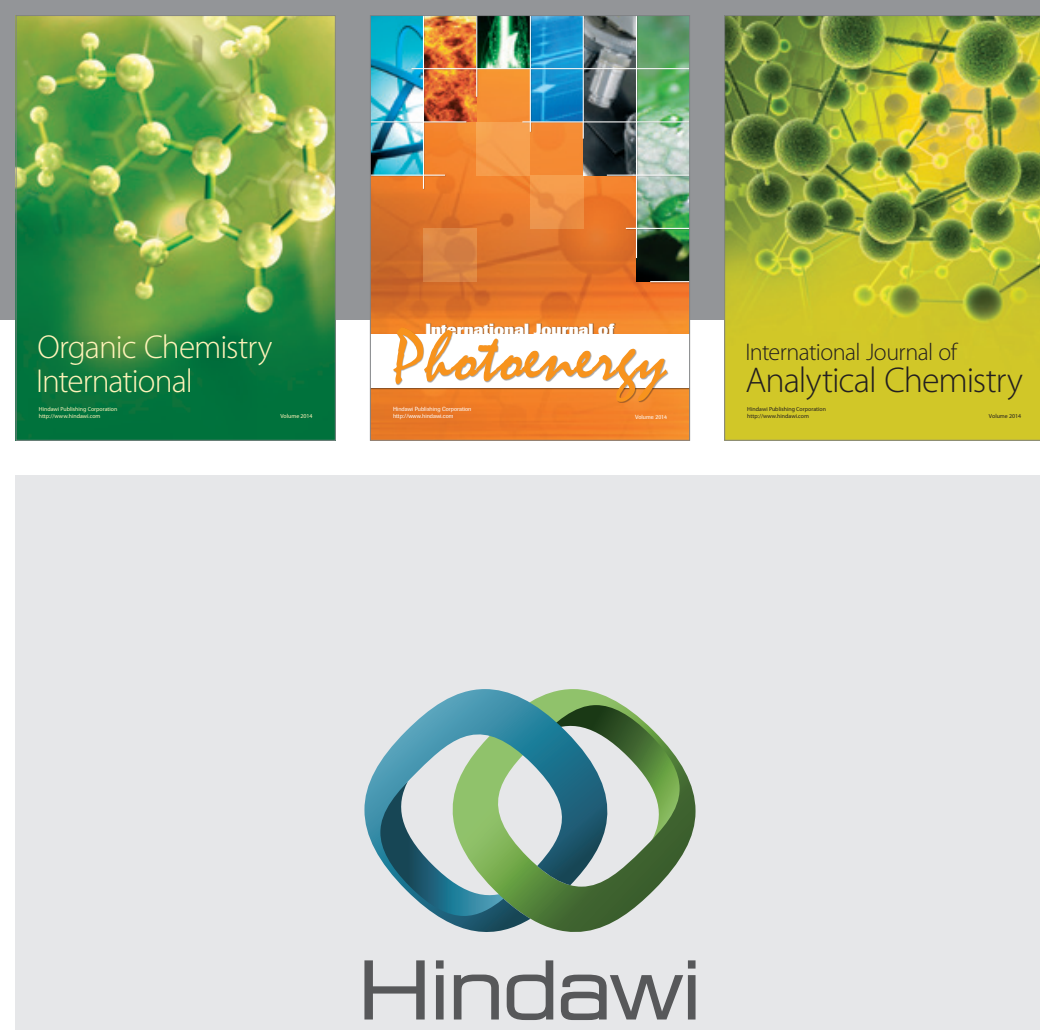

Submit your manuscripts at

http://www.hindawi.com
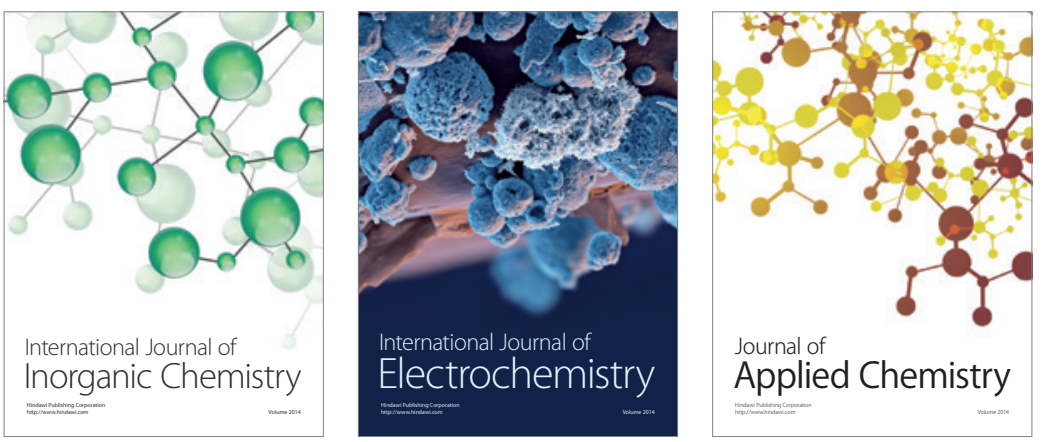

Journal of

Applied Chemistry
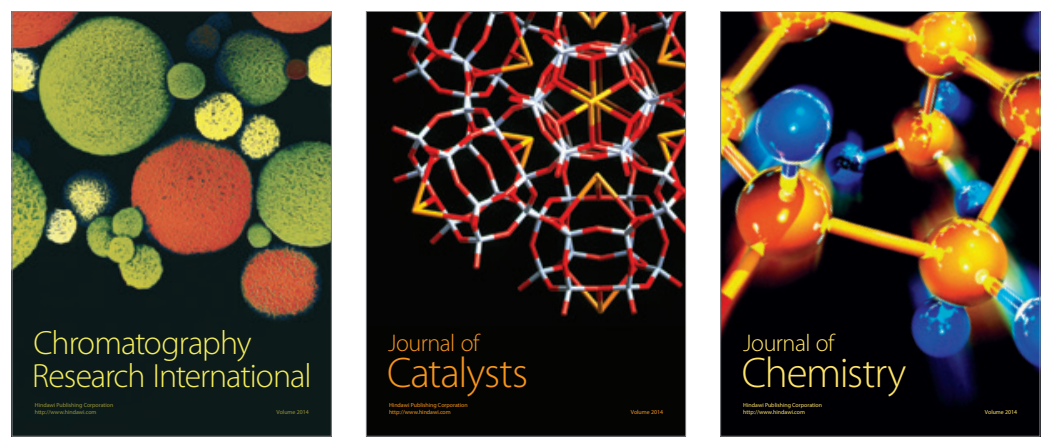
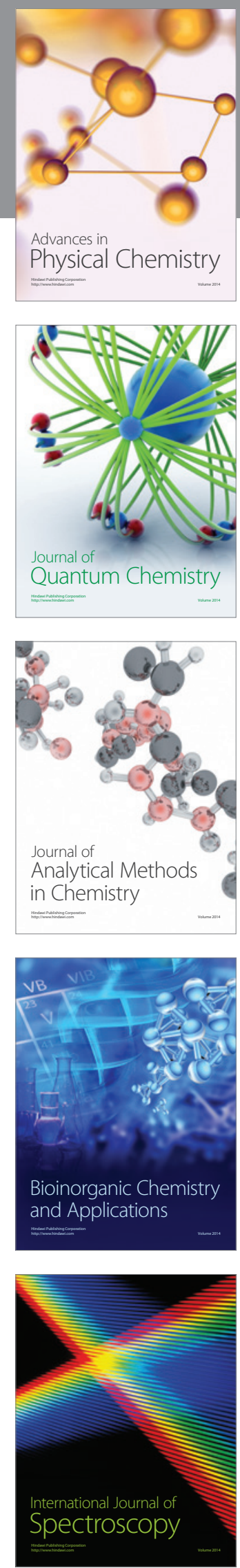